\title{
Schlaganfallprävention in der klinischen Praxis
}

\author{
Neue Daten zum PFO- und LAA-Okkluder
}

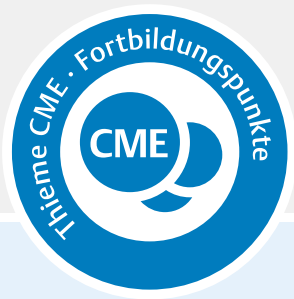

Johannes Jakob Hartung ${ }^{1,2}$, Carsten Skurk ${ }^{1,2}$, David Manuel Leistner ${ }^{1,2,3}$, Ulf Landmesser ${ }^{1,2,3}$

1 Medizinische Klinik für Kardiologie, Charité - Universitätsmedizin Berlin, Campus Benjamin Franklin, Berlin

2 Deutsches Zentrum für Herz-Kreislauf-Forschung (DZHK), Standort Berlin, Berlin

3 Berlin Institute of Health (BIH), Berlin

Bibliografie

10.1055/a-0605-7652

\section{ZUSAMMENFASSUNG}

Zur Prophylaxe kardioembolischer Schlaganfälle und Thromboembolien hat die Implantation intrakardialer Okkluder sowohl bei Patienten mit Vorhofflimmern als auch bei Patienten mit persistierendem Foramen ovale (PFO) im Vergleich zu einer lebenslangen antithrombotisch-medikamentösen Therapie in den letzten Jahren zunehmend an Bedeutung in der klini- schen Praxis gewonnen. Neue Daten aus großen Studien, die die Katheter-basierten Systeme zum Verschluss des linken Vorhofohres (left atrial appendage, kurz: LAA) bei Patienten mit Vorhofflimmern und relevantem Schlaganfallrisiko hinsichtlich Ihrer Effektivität und Sicherheit randomisiert im Vergleich zu einer medikamentösen Therapie mit Vitamin-K-Antagonisten untersucht haben, zeigen vielversprechende Ergebnisse. Darüber hinaus konnte in 3 randomisierten Studien, die im vergangenen Jahr im New England Journal of Medicine publiziert wurden, eine signifikante Überlegenheit des interventionellen PFO-Verschlusses im Vergleich zu einer alleinigen medikamentösen Therapie bei Patienten < 60 Jahre mit kryptogenem Schlaganfall nachgewiesen werden. Bislang nicht untersucht ist der randomisierte Vergleich der LAA-Verschlusssysteme mit einer NOAK-Therapie. Auch zur Beantwortung der Frage nach der optimalen, risikoadaptieren, medikamentösen post-LAAOkkluder-Implantationstherapie müssen die Ergebnisse aktuell rekrutierender Studien abgewartet werden.

\section{Einleitung}

Kardiale Embolien werden als eine wesentliche Ursache von Schlaganfällen (bei ca. 25\%) angesehen [1]. Bei jüngeren Patienten wird insbesondere ein persistierendes Foramen ovale (PFO) als eine mögliche Ursache für cerebrovaskuläre Embolien seit einigen Jahren intensiv diskutiert. Im letzten Jahr wurden 3 wichtige randomisierte Studien zu diesem Thema im New England Journal of Medicine publiziert [2-4]. Bei älteren Patienten wird insbesondere das Vorhofflimmern und eine damit einhergehende kardiale Thrombenbildung als eine wichtige kardioembolische Schlaganfallursache angesehen. Zur Prävention von Schlaganfällen und peripheren Embolien bei Patienten mit Vorhofflimmern besteht in Abhängigkeit vom $\mathrm{CHA}_{2} \mathrm{DS}_{2}$ VASc-Score nach den aktuellen Leitlinien der europäischen Gesellschaft für Kardiologie (ESC) eine Indikation zur dauerhaften Antikoagulation [5]. Unter einer Antikoagulation muss allerdings mit einer erhöhten Inzidenz von Blutungskomplikationen gerechnet werden. Diese liegt, in Abhängigkeit von weiteren Begleiterkrankungen, zwischen $1 \%$ und mehr als $10 \%$ pro Jahr $[6,7]$. Bei Patienten mit deutlich erhöhtem Blutungsrisiko unter einer Antikoagulation findet seit einigen Jahren die Implantation Katheter-basierter Okkluder zum Verschluss des linken Vorhofohres (left atrial appendage, kurz: LAA) Anwendung, allerdings werden hier weitere Studien benötigt, welche nun initiiert werden (z. B. CLOSURE-AF-Studie).
Die aktuelle Datenlage und klinische Anwendung in Hinsicht auf die Patientenselektion soll für den Katheterbasierten PFO- und LAA-Verschluss im Folgenden kurz dargestellt werden.

\section{Daten zum PFO-Okkluder}

Bei ca. $30 \%$ der jungen Schlaganfallpatienten kann trotz ausführlicher Abklärung keine Schlaganfall-Ursache eruiert werden. Ein PFO ist bei ca. 50\% dieser Patienten nachweisbar, während die Inzidenz in der Normalpopulation etwa $25 \%$ beträgt [8].

Seit über 20 Jahren wird der perkutane Verschluss eines PFO zur Sekundärprophylaxe bei jüngeren Patienten mit kryptogenem Schlaganfall kontrovers diskutiert. Die initiale Datenlage war durch mehrere inkonklusive Studien gekennzeichnet. In dem 2013 von Meier et al. publizierten, randomisierten und kontrollierten PC-Trial [9] wurden 414 Patienten mit einem Amplatzer PFO-Okkluder oder einer konservativen medikamentösen Therapie über durchschnittlich 4 Jahre behandelt. Insgesamt 7 Patienten in der Verschluss-Gruppe $(3,4 \%)$ und 11 in der Gruppe mit einer medikamentösen Therapie $(5,2 \%)$ verstarben oder zeigten eine TIA, einen nicht-tödlichen Schlaganfall oder eine periphere Embolie $(H R=0,63 ; \mathrm{Cl} 0,24-1,62$; $P=0,34)$. Nicht-tödliche Schlaganfälle wurden bei einem 
Patienten $(0,5 \%)$ in der Okkluder-Gruppe und 5 Patienten (2,4\%) mit einer medikamentösen Therapie beobachtet $(\mathrm{HR}=0,20 ; \mathrm{Cl} 0,02-1,72 ; \mathrm{P}=0,14)$. Auch die ebenfalls 2013 veröffentlichten CLOSURE-I-Studie [10], die 909 Patienten mit einem STARFlex Septal Closure-System oder medikamentös therapierte Patienten über 2 Jahre beobachtete, konnte keinen signifikanten Unterschied im primären Endpunkt aus Schlaganfall, TIA und systemischer Embolie (5,5\% und 6,8\%; $P=0,37$ ) über diesen relativ kurzen Zeitraum nachweisen. Im RESPECT-Trial, der dritten randomisierten Studie, zeigte sich nach einer kurzen durchschnittlichen Beobachtungsperiode von 2,1 Jahren in der Intention-to-treat-(ITT)-Analyse kein signifikanter Vorteil des PFO-Verschlusses im Vergleich mit einer medikamentösen Therapie (Aspirin, Warfarin, Clopidogrel oder Aspirin und Dipyridamol). Allerdings waren hier Signale für einen Vorteil des PFO-Okkluders in der nach Protokoll therapierten Patientenpopulation nachweisbar. Zusammenfassend konnte in den 3 initialen randomisierten Studien bei Patienten nach stattgehabtem kryptogenem Schlaganfall und nachgewiesenem PFO durch die Implantation eines PFO-Okkluders keine signifikante Risikoreduktion in der Sekundärprävention zerebro-embolischer Ereignisse oder in Hinsicht auf eine verringerte Mortalität im Vergleich zu einer medikamentösen Therapie beobachtet werden.

\section{Neue randomisierte Daten zum PFO-Okkluder}

Die nun vorliegenden Langzeit-Beobachtungsdaten der RESPECT-Studie zeigen einen signifikanten Vorteil der PFOOkkluder-Implantation. In dieser Studie [2] wurden 980 Patienten zwischen 18 und 60 Jahren mit PFO und Z. n. kryptogenem Schlaganfall eingeschlossen. Die Patienten wurden randomisiert mit einem interventionellen PFOVerschluss oder einer ausschließlich medikamentösen antithrombotischen Therapie behandelt (überwiegend Aspirin). Nach einem deutlich längeren mittleren Followup von ca. 6 Jahren, zeigte sich nun in der ITT-Analyse ein deutlicher Vorteil in der PFO-Verschlussgruppe. Bei 18 Patienten der PFO-Okkludergruppe kam es im Verlauf zu einem erneuten ischämischen Schlaganfall $(0,58$ Ereignisse/100 Patientenjahre), während dies in der medikamentösen Gruppe bei 28 Patienten (1,07 Ereignisse/100 Patientenjahre) ( $H R=0,55 ; C l 0,31-0,999 ; P=0,046)$ beobachtet wurde.

In der ebenfalls im vergangenen Jahr publizierten REDUCE-Studie [3] wurden 664 Patienten mit PFO und kryptogenem Schlaganfall im Verhältnis 2:1 zu einer interventionellen Therapie mit einem PFO-Okkluder oder zu einer alleinigen antithrombozytären Therapie randomisiert. Für den kombinierten primären Endpunkt wurde das Auftreten eines erneuten symptomatischen oder eines klinisch stummen (Nachweis in der zerebralen Bildgebung) Hirninfarkts innerhalb von 24 Monaten nach Randomisierung bewertet. Während eines mittleren Follow-up-Zeitraums von 3,2 Jahren wurden mit 6 Schlaganfällen $(1,4 \%)$ in der
PFO-Okkluder-Gruppe und 12 Schlaganfällen $(5,4 \%)$ in der medikamentösen Gruppe $(\mathrm{HR}=0,23$; Cl 0,09-0,62; $P=0,002)$, d. h. signifikant weniger symptomatische Hirninfarkte nach PFO-Verschluss beobachtet.

In der CLOSE-Studie [4] wurden 663 Patienten zwischen 16 und 60 Jahren, die einen kryptogenen Schlaganfall erlitten hatten und bei denen aufgrund eines assoziierten Vorhofseptumaneurysmas oder eines großen interatrialen Shunts ein PFO als ursächlich für den Schlaganfall angenommen wurde, im Verhältnis 1:1:1 zu einem interventionellen PFO-Verschluss, einer alleinigen anti-thrombozytären Therapie oder einer oralen Antikoagulation randomisiert. Primärer Endpunkt war das Auftreten eines Schlaganfalls. Keiner der Patienten der PFO-Okkludergruppe erlitt während der Nachbeobachtungsperiode einen Schlaganfall. Damit war die Rate für das Auftreten eines erneuten Schlaganfalls nach PFO-Verschluss signifikant niedriger, als in der Gruppe, die eine alleinige antithrombozytäre Therapie erhielt ( 14 Schlaganfälle, $\mathrm{HR}=0,03 ; \mathrm{Cl}$ 0,16-0,82). In der Patientengruppe mit einer oralen Antikoagulation traten 3 Schlaganfälle auf.

Zusammenfassend belegen die neuen Daten eine signifikante Reduktion des Risikos für einen erneuten Schlaganfall bei jüngeren Patienten mit kryptogenem Schlaganfall nach PFO-Verschluss verglichen mit einer konservativ-medikamentösen Therapie in der Sekundärprophylaxe. Die Prozedur kann heute sicher und mit sehr geringem Risiko mit getesteten PFO-Okkludern durchgeführt werden. Die aktuellen Daten werden sicher in den neuen Guidelines zu Änderungen in den Empfehlungen führen.

\section{Patientenselektion - Einsatz des Katheter- basierten PFO-Verschlusses in der klinischen Praxis}

Der klinische Stellenwert des interventionellen PFO-Verschlusses bei Patienten mit Z. n. kryptogenem Schlaganfall unter Einbeziehung der aktuellen Datenlage ist in $\mathbf{A b b} \mathbf{~} \mathbf{1}$ dargestellt. Für die Versorgung mit einem PFO-Okkluder sollten die Ein- und Ausschlusskriterien der randomisierten Studien beachtet werden.

Laut aktueller Datenlage gibt es heute gute Evidenz, dass insbesondere Patienten mit einem Alter bis 60 Jahre und kryptogenem Schlaganfall von einem interventionellen PFO-Verschluss hinsichtlich der Reduktion des Risikos eines erneuten Schlaganfalls profitieren, besonders bei Hinweisen für einen mittleren oder großen Shunt über das PFO.

Die Größe des PFO wird über das Shuntvolumen (spontaner Rechts-Links- oder bidirektionaler Shunt) im Farb/ cw-Doppler oder nach Provokationsmanöver mittels TEE dargestellt. Auch das Vorhandensein eines Vorhofseptumaneurysmas (Auslenkung in der 2D-Echokardiografie $>10 \mathrm{~mm}$ ) gilt als ein mögliches Kriterium für ein er- 


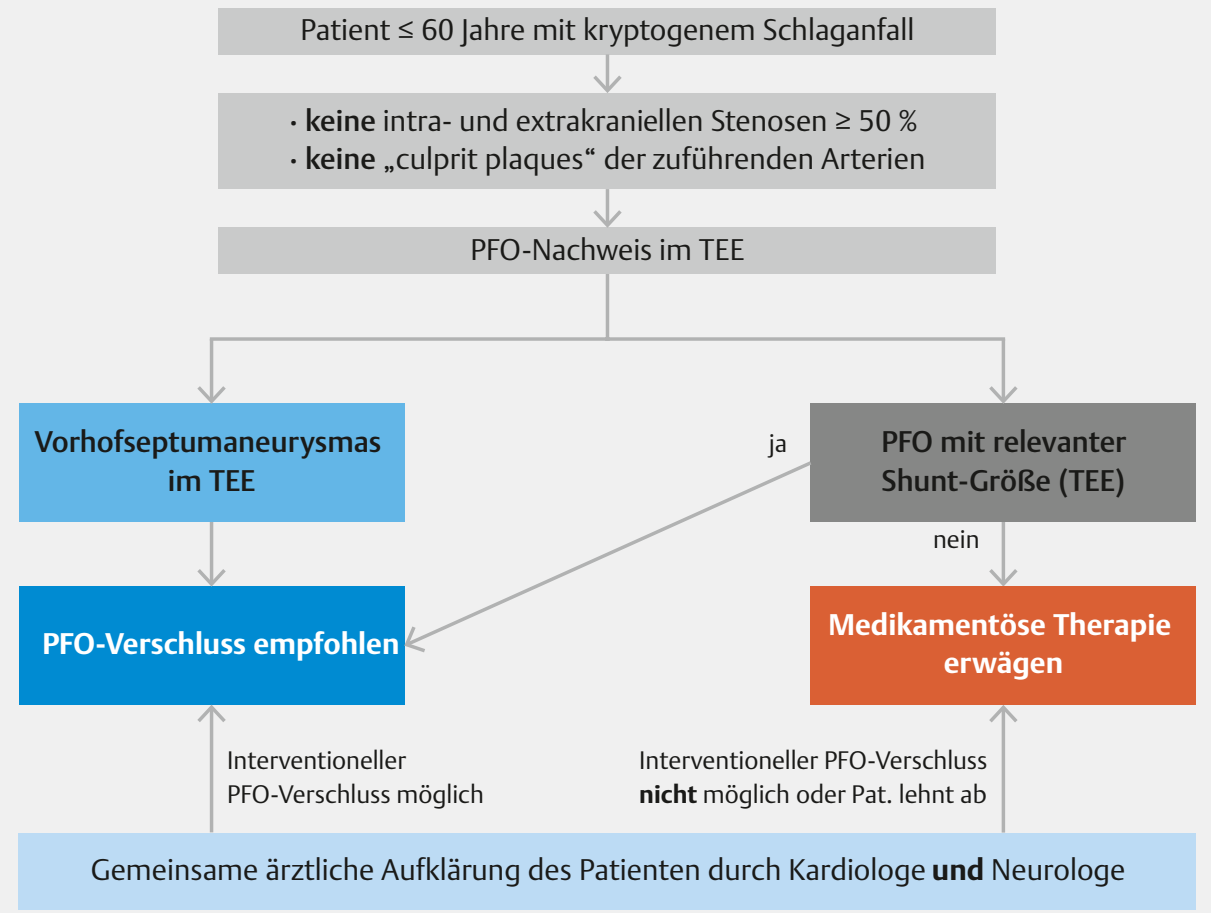

- Abb. 1 Algorithmus der Schlaganfallprävention bei Patienten mit persistierendem Foramen ovale (PFO) [rerif].

höhtes Risiko. Die Abklärung der Genese des Schlaganfalls umfasst den Ausschluss von signifikanten Stenosen der hirnversorgenden sowie intrakraniellen Arterien u. a. anhand des CT-morphologischen Bildes.

\section{Daten zum LAA-Okkluder}

Bislang wurden 2 randomisierte, kontrollierte Studien publiziert, die den katheterinterventionellen Verschluss des linken Vorhofohres im Vergleich zu einer Antikoagulation mit einem Vitamin-K-Antagonisten untersucht haben (PROTECT-AF [11], PREVAIL [12]). In der PROTECTAF-Studie wurde der interventionelle Vorhofohrverschluss mit dem Watchman ${ }^{\mathrm{TM}}{ }_{\text {-OKkluder bei }} 707$ Patienten mit Vorhofflimmern und relevantem Schlaganfallrisiko nach 2:1-Randomisierung im Vergleich zu Warfarin getestet. Für den LAA-Okkluder konnten nach einer mittleren Nachbeobachtungszeit von 3,8 Jahren sowohl die Kriterien für eine Nichtunterlegenheit (A-posteriori-Wahrscheinlichkeit $>99,9 \%$ ), als auch für eine Überlegenheit (A-posteriori-Wahrscheinlichkeit $96 \%$ ) bezüglich des kombinierten primären Endpunkts aus Schlaganfall, systemischer Embolie und kardiovaskulärer Mortalität erreicht werden (2,3 Ereignisse/100 Patientenjahre für die LAA-Okkluder-Gruppe versus 3,8 Ereignisse/100 Patientenjahre für die Warfarin-Gruppe; $H R=0,60 ; C l$ 0,41-1,05). Zudem war der LAA-Verschluss mittels Watchman ${ }^{\mathrm{TM}}$-Okkluder sowohl hinsichtlich der Gesamtmortalität (3,2 Ereignisse/100 Patientenjahre in der LAA-Okkluder-Gruppe versus 4,8 Ereignisse/100 Patientenjahre in der Warfarin-Gruppe; $H R=0,66$;
Cl 0,45-0,98), als auch hinsichtlich der kardiovaskulären Mortalität (1,0 Ereignisse/100 Patientenjahre in der LAAOkkluder-Gruppe versus 2,4 Ereignisse/100 Patientenjahre in der Warfarin-Gruppe; $\mathrm{HR}=0,40 ; \mathrm{Cl} 0,21-0,75)$ nach 3,8 Jahren Beobachtungszeit einer oralen Antikoagulation überlegen [13].

In der PREVAIL-Studie wurden 407 Patienten 2:1 zu einem interventionellen Vorhofohrverschluss mittels Watchman ${ }^{\mathrm{TM}}$-Okkluder oder Warfarin-Therapie randomisiert. Die Studie verfehlte nach 18 Monaten follow-up den primären Effektivitätsendpunkt (Schlaganfall, kardiovaskulärer Tod und systemische Embolie) bei einer unerwartet niedrigen Ereignisrate in der Antikoagulationsgruppe (2,9\% in der Kontrollgruppe versus 5,9\% in der OkkluderGruppe) [12]. In einer von Holmes et al. veröffentlichten Metaanalyse, die die Ergebnisse der PROTECT-AF- und PREVAIL-Studie zusammenfasst, wurde eine Nichtunterlegenheit des LAA-Verschlusses für Schlaganfall, systemische Embolie und kardiovaskulären $\operatorname{Tod}(\mathrm{HR}=0,79 ; \mathrm{Cl} 0,53-1,2)$ beobachtet [14], wobei die Zahl der Patienten in beiden Studien noch relativ limitiert war.

Die zunehmende Erfahrung bei der Implantation bestimmter LAA-Okkludersysteme (Watchman ${ }^{\mathrm{TM}}$ und Amplatzer ${ }^{\mathrm{TM}}$ Amulet ${ }^{\mathrm{TM}}$ ) spiegelt sich auch in den aktuellen großen multizentrischen Registerdaten (EWOLUTION-Register [15] und Global-Amulet-Register [16]) wider, die den klinischen Einsatz des Watchman ${ }^{\mathrm{TM}}$-Okkluders bzw. des Amplatzer ${ }^{\mathrm{TM}}$ Amulet ${ }^{\mathrm{TM}}$ Devices bei Hochrisikopatienten erfassen. In bei- 
den Registern zeigte sich eine sehr hohe Implantationserfolgsrate und ein niedriges Risiko für das Auftreten periprozeduraler Komplikationen. Die periprozedurale Sicherheit konnte durch eine verbesserte Implantationstechnik und die Weiterentwicklung der Devices signifikant verbessert werden $[15,16]$. Weitere LAA-Okkluder sind in der klinischen Entwicklung.

In den aktuellen ESC-Guidelines ist der interventionelle Verschluss des linken Vorhofohres mittels Okkluder bei Patienten mit Kontraindikation für eine orale Antikoagulation mit einer Ilb-Indikation angegeben [5].

\section{Neue Studien zum LAA-Verschluss}

Bislang existieren noch keine randomisierten Daten zum direkten Vergleich des perkutanen LAA-Verschlusses mit einer medikamentösen NOAK-Therapie bei Patienten mit hohem Schlaganfall- und hohem Blutungsrisiko. Aus diesem Grund startete im Februar 2018 mit der CLOSUREAF-Studie (ClinicalTrials.gov Identifier: NCT03463317) die bislang weltweit größte geplante, randomisierte, kontrollierte Studie, die bei 1512 Patienten mit Vorhofflimmern und hohem Schlaganfall- $\left(\mathrm{CHA}_{2} \mathrm{DS}_{2}\right.$ VASc-Score $\left.\geq 2\right)$ und hohem Blutungsrisiko (stattgehabte Blutung BARC-Typ $\geq 3$, HAS-BLED-Score $\geq 3$ oder chronischer Niereninsuffizienz mit einer eGFR zwischen $15-30 \mathrm{ml} / \mathrm{min} / 1,73 \mathrm{~m}^{2}$ ) in einem Therapiestrategie-Vergleich die Sicherheit und Effektivität der ausschließlich medikamentösen Antikoagulation mit einem interventionellen Verschluss des linken Vorhofohres, randomisiert im Verhältnis 1:1 (LAA-Okkluder versus „best medical care“) vergleicht. In der „best medical care“Gruppe können alle zur Anwendung in Deutschland zugelassenen NOAK eingesetzt werden, wenn keine Kontraindikationen vorliegen. Die postinterventionelle Therapie nach LAA-Verschluss umfasst eine zeitlich limitierte duale Thrombozytenaggregationshemmung. Der primäre kombinierte Endpunkt („net clinical benefit“) ist definiert als die Überlebenszeit frei von Schlaganfall (ischämisch oder hämorrhagisch), systemischer Embolie, schwerer Blutung (BARC Typ 3-5) und kardiovaskulärem oder unklarem Tod. Die CLOSURE-AF-Studie wird wertvolle Hinweise für den klinischen Einsatz der LAA-Okkluder bei Hochrisikopatienten mit Vorhofflimmern geben.

In der STROKECLOSE-Studie (ClinicalTrials.gov Identifier: NCT02830152) sollen insgesamt 750 Patienten mit Vorhofflimmern und hohem Schlaganfallrisiko $\left(\mathrm{CHA}_{2} \mathrm{DS}_{2}\right.$ VASc-Score $\geq 2$ ) sowie einem klinischen und bildmorphologischen Nachweis (CT/MRT) einer intrazerebralen Blutung (ICB) innerhalb der letzten 6 Monate, im Verhältnis 2:1 auf einem LAA-Okkluder oder zu einer „best medical care“-Therapie (inkl. NOAK) randomisiert werden. In der Okkluder-Gruppe ist ausschließlich das Amplatzer ${ }^{\mathrm{TM}}$ Amulet ${ }^{\mathrm{TM}}$ Device zugelassen. Primäre Endpunkte sind die Gesamtmortalität sowie ein kombinierter Endpunkt, bestehend aus Schlaganfall (ischämische oder hämorrha- gische), systemischer Embolie und lebensbedrohlicher oder ausgedehnter Blutungen.

In der PRAGUE-17-Studie werden insgesamt 396 Patienten eingeschlossen, die neben einem Vorhofflimmern mindestens eines der folgenden Kriterien erfüllen: stattgehabte signifikante Blutung, stattgehabtes kardio-embolisches Ereignis oder $\mathrm{CHADS}_{2}$-Score $\geq 2$ oder $\mathrm{CHA}_{2} \mathrm{DS}_{2}$ VAScScore $\geq 3$. Die Randomisierung erfolgt im Verhältnis $1: 1$ zwischen LAA-Okkluder (Amplatzer ${ }^{\mathrm{TM}}$ Amulet $^{\mathrm{TM}}$ und Watchman ${ }^{\mathrm{TM}}$ ) und medikamentöser NOAK-Therapie. Als primäerer kombinierter Endpunkt ist das Auftreten eines der folgenden Ereignisse innerhalb von 24 Monaten nach Randomisierung definiert: Schlaganfall oder TIA, systemisch kardioembolisches Ereignis, klinisch signifikante Blutung, kardiovaskulär bedingter Tod und signifikante periprozedurale oder Device-assoziierte Komplikation [17].

\section{Patientenselektion - Einsatz des Katheter- basierten LAA-Verschlusses in der klinischen Praxis}

Die aktuellen Guidelines der Europäischen Gesellschaft für Kardiologie [5] und ein EHRA/EAPCI „consensus document" empfehlen einen LAA-Verschluss bei Patienten mit Vorhofflimmern und hohem Schlaganfallrisiko, welche Kontraindikationen für eine Langzeit-(N)OAK-Therapie oder ein hohes Blutungsrisiko aufweisen, zu erwägen. Hierbei handelt es sich in erster in erster Linie um folgende Patienten:

- stattgehabte Blutungsereignisse wie nicht-traumatische intrakranielle Blutungen, z. B. Blutungen aufgrund einer zerebralen Amyloid-Angiopathie oder lobuläre Blutungen (Bleeding Academic Research Consortium [BARC] Definition Typ 3c)

- schwere Blutungen BARC Typ 3a oder 3b: gastrointestinale, urogenitale oder Atemwegstrakt-Blutungen mit persistierendem erhöhten Blutungsrisiko, die Ursache der Blutung kann nicht behoben werden.

- erheblich erhöhtes Blutungsrisiko unter Antikoagulation.

\section{Fazit}

Die aktuellen Studien zeigen für den PFO-Verschluss überzeugende Ergebnisse hinsichtlich der Effektivität zur Reduktion des Schlaganfallrisikos bei jüngeren Patienten (<60 Jahre) nach kryptogenem Schlaganfall.

Zur Schlaganfallprophylaxe bei Vorhofflimmern konnte die Nichtunterlegenheit der LAA-Okkluder im Vergleich zu einer Antikoagulation mit Vitamin-K-Antagonisten in einer randomisierten, kontrollierten Studie beobachtet werden, bei allerdings noch begrenzter Patientenzahl in der Studie. Hinsichtlich der kardiovaskulären Mortalität zeigte sich im Vergleich zu Vitamin-K-Antagonisten nach 4 Jahren Beobachtungszeit ein möglicher Vorteil der interventionellen Therapie. Die Ergebnisse neuer Studien, 
die die Okkluder randomisiert mit einer medikamentösen Therapie mit nicht-Vitamin-K-abhängigen Antikoagulanzien (NOAK) bei Patienten mit Vorhofflimmern und hohem Blutungsrisiko untersuchen, bleiben abzuwarten.

Interessenkonflikt
J.H.: kein Interessenkonflikt
C.S.: kein Interessenkonflikt
D.L.: kein Interessenkonflikt
U. L.: has received lecture or advisory fees from Abbott and Boston Scientific

\section{Korrespondenzadresse}

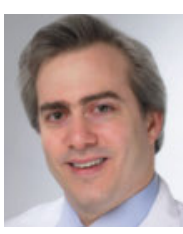

\section{Univ.-Prof. Dr. med. Ulf Landmesser}

BIH Professor für Kardiologie

Ärztliche Leitung Charité Centrum für Herz-, Kreislauf- und Gefäßmedizin

Direktor der Medizinischen Klinik für

Kardiologie

Charité - Universitätsmedizin Berlin

Campus Benjamin Franklin

Hindenburgdamm 30

12203 Berlin

ulf.landmesser@charite.de

\section{Literatur}

[1] Dietl M, Pohle R, Weingartner M et al. [Stroke etiology and long-term need of care in ischemic stroke patients]. Fortschr Neurol Psychiatr 2009; 77: 714-719

[2] Saver JL, Carroll JD, Thaler DE et al. Long-Term Outcomes of Patent Foramen Ovale Closure or Medical Therapy after Stroke. New Engl J Med 2017; 377: 1022-1032

[3] Sondergaard L, Kasner SE, Rhodes JF et al. Patent Foramen Ovale Closure or Antiplatelet Therapy for Cryptogenic Stroke. New Engl J Med 2017; 377: 1033-1042

[4] Mas JL, Derumeaux G, Guillon B et al. Patent Foramen Ovale Closure or Anticoagulation vs. Antiplatelets after Stroke. New Engl J Med 2017; 377: 1011-1021

[5] Kirchhof P, Benussi S, Kotecha D et al. 2016 ESC Guidelines for the management of atrial fibrillation developed in collaboration with EACTS. Eur Heart J 2016; 37: 2893-2962
[6] Pisters R, Lane DA, Nieuwlaat $R$ et al. A novel user-friendly score (HAS-BLED) to assess 1 -year risk of major bleeding in patients with atrial fibrillation: the Euro Heart Survey. Chest 2010; 138: 1093-1100

[7] Lip GY, Frison L, Halperin JL, Lane DA. Comparative validation of a novel risk score for predicting bleeding risk in anticoagulated patients with atrial fibrillation: the HAS-BLED (Hypertension, Abnormal Renal/Liver Function, Stroke, Bleeding History or Predisposition, Labile INR, Elderly, Drugs/Alcohol Concomitantly) score. J Am Coll Cardiol 2011; 57: 173-180

[8] Messe SR, Kent DM. Still no closure on the question of PFO closure. New Engl J Med 2013; 368: 1152-1153

[9] Meier B, Kalesan B, Mattle HP et al. Percutaneous closure of patent foramen ovale in cryptogenic embolism. New Engl J Med 2013; 368: 1083-1091

[10] Furlan A], Investigators CI. PFO Closure: CLOSURE. Stroke 2013; 44: 45-47

[11] Holmes DR, Reddy VY, Turi ZG et al. Percutaneous closure of the left atrial appendage versus warfarin therapy for prevention of stroke in patients with atrial fibrillation: a randomised non-inferiority trial. Lancet 2009; 374: 534-542

[12] Holmes DR, Jr., Kar S, Price M] et al. Prospective randomized evaluation of the Watchman Left Atrial Appendage Closure device in patients with atrial fibrillation versus long-term warfarin therapy: the PREVAIL trial. J Am Coll Cardiol 2014; 64: $1-12$

[13] Reddy VY, Sievert H, Halperin J et al. Percutaneous left atrial appendage closure vs warfarin for atrial fibrillation: a randomized clinical trial. Jama 2014; 312: 1988-1998

[14] Holmes DR, Jr., Doshi SK, Kar S et al. Left Atrial Appendage Closure as an Alternative to Warfarin for Stroke Prevention in Atrial Fibrillation: A Patient-Level Meta-Analysis. J Am Coll Cardiol 2015; 65: 2614-2623

[15] Boersma LV, Ince H, Kische $\mathrm{S}$ et al. Efficacy and safety of left atrial appendage closure with WATCHMAN in patients with or without contraindication to oral anticoagulation: 1-Year follow-up outcome data of the EWOLUTION trial. Heart rhythm 2017; 14: 1302-1308

[16] Landmesser U, Schmidt B, Nielsen-Kudsk JE et al. Left atrial appendage occlusion with the AMPLATZER Amulet device: periprocedural and early clinical/echocardiographic data from a global prospective observational study. Eurolntervention 2017; 13: 867-876

[17] Osmancik P, Tousek P, Herman D et al. Interventional left atrial appendage closure vs novel anticoagulation agents in patients with atrial fibrillation indicated for long-term anticoagulation (PRAGUE-17 study). Am Heart J 2017; 183: $108-114$ 